\title{
CAÍdA DE PRESIÓN EN LECHO FIJO DE CUBOS DE PAPA, REMOLACHA Y ZANAHORIA CONSIDERANDO ENCOGIMIENTO'
}

\author{
Javier TELIS-ROMERO ${ }^{2, *}$, Paulo José do Amaral SOBRAL ${ }^{3}$
}

\begin{abstract}
RESUMEN
En este trabajo fue determinada experimentalmente la porosidad de lechos de cubos de papa, remolacha y zanahoria en función de la humedad con aristas iniciales de $8 \mathrm{~mm}$. La porosidad del lecho, con diferentes contenidos de humedad obtenida por secado a $70^{\circ} \mathrm{C}$ por diferentes tempos, fue calculada utilizando las determinaciones experimentales de volumen del lecho y volumen real de las partículas. Estos dos volumenes fueran bien representados por ecuaciónes lineares basadas en la Ley de la aditividad de los volumens. La porosidad fue representada satisfactoriamente por una ecuación parabólica. La relación volumen del lecho adimensional y humedad fue representada por polinomios de tercer grado. Utilizando un equipamiento con sistema de control, fue determinada experimentalmente la caída de presión a través de un lecho fijo de cubos de papas, zanahoria y remolacha de 100mm de espesura y los resultados fueron representados de manera satisfactoria por una ecuación obtenida por una modificación empírica del modelo de Ergun. Como era de esperarse, la caída de pression aumento com la disminución de la porosidad del lecho, consequencia del encogimiento de los productos.

Palabras-clave: vegetales; secado; encogimiento; caída de presión.
\end{abstract}

\section{RESUMO}

QUEDA DE PRESSÃO EM LEITO FIXO DE CUBOS DE BATATA, BETERRABA E CENOURA CONSIDERANDO ENCOLHIMENTO. NESTE trabalho, o porosidade de leitos de cubos de batata, beterraba e cenoura com arestas iniciais de $8 \mathrm{~mm}$, foi determinada experimentalmente em função da umidade. A porosidade do leito, com diferentes umidades, obtidas por secagem a $70^{\circ} \mathrm{C}$ por tempos diferentes, foi calculada utilizando-se as determinações experimentais de volume do leito e volume real das partículas. Esses dois volumes foram bem representados por equações lineares baseadas na lei da adtividade dos volumes. A porosidade foi representada satisfatoriamente por uma equação parabólica. A relação entre o volume do leito admensional e a umidade foram representadas por polinômios de terceiro grau. A queda de pressão através de um leito fixo de cubos de batata, beterraba e cenoura de $100 \mathrm{~mm}$ de espessura foi determinada experimentalmente utilizando-se um equipamento com sistema de controle. Os resultados foram representados de maneira satisfatória por uma equação desenvolvida empiricamente a partir do modelo de Ergun. Como era de se esperar, a queda de pressão aumentou com a diminuição da porosidade do leito, conseqüência do encolhimento dos produtos.

Palavras-chave: vegetais; secagem; encolhimento; queda de pressão.

\section{1 - INTRODUCCIÓN}

La deshidratación constituye una etapa fundamental en el procesamiento, almacenamiento y conservación de muchos productos alimenticios naturales como frutas, vegetales y granos. En general este proceso implica, a menos que el material haya sido sometido a un pretratamiento que destruya la estructura celular, la deshidratación de tejidos celulares en funcionamiento. A medida que avanza la deshidratación, las células evolucionan desde una estructura viva altamente organizada hasta un conjunto desordenado de los constituyentes fundamentales, debido a la deformación del material [3]. Así, los mecanismos y propiedades de transporte resultan fuertemente dependientes de la naturaleza del material, su contenido de humedad y su temperatura, y consecuentemente, de su cambio de dimensiones.

Desde el punto de vista del fenómeno de transporte y la simulación del proceso, el secado de alimentos es un problema de transporte simultáneo de materia y

1. Recebido para publicação em 06/06/2002. Aceito para publicação em 06/08/2003 (000848).

2. Universidade Estadual Paulista Campus São José do Rio Preto - Instituto de Biociências, Letras e Ciências Exatas - Rua Cristóvão Colombo, 2265 Jd. Nazareth CEP 15054-000.E-mail: javier@eta.ibilce.unesp.br

3. Departamento de Zootecnia e Engenharia de Alimentos, Universidade de São Paulo (USP) CEP 13635-900, Caixa Postal 23, Pirassununga - SP. * A quem a correspondência deve ser enviada. energía, con la dificultad adicional de que el material sufre importantes cambios estructurales, fisicoquimicos y biológicos durante el proceso. Por otro lado, en el diseño de secadores, un proyecto básico es el cálculo de la presión que el soplador necesita para vencer la caída de presión que un lecho provoca en el flujo de aire. Cuando el aire es forzado a desplazarse a través de los intersticios existentes entre las particulas que constituyen un lecho poroso, existe una resistencia ofrecida por el lecho que implica en una caída de presión, causada por la disipación de energía motivada por la turbulencia y fricción del fluido. Algunos factores combinados son los que causan esta caída de presión a través del lecho de particulas alimenticias. Los factores que contribuyen incluyen el caudal de aire, características como porosidad, esfericidad, tamaño y distribución de tamaño de las partículas, configuración de los espacios y espesura del lecho de partículas [6]. Por lo tanto, para este calculo en un secador donde las partículas encogen cuando están siendo deshidratadas, es necesario conocer la relación entre porosidad, diámetro de la partícula, esfericidad y humedad del lecho de particulas.

Varios trabajos sobre encogimiento de productos alimenticios tales como uvas [17], champiñones [2], papas [4, 10, 16, 18, 20], yuca [1], ajo [9], frutas y legumbres diversos $[7,8,19,21]$ durante el secado, poden ser encontrados en la literatura. Pero, pocos son los trabajos sobre el encogimiento del lecho de estos productos durante el secado [15]. 
La perdida por fricción para flujo a través de lechos rellenos puede expresarse por la ecuación de Ergun (Ecuación 1), basada en la hábil combinación de la ecuación de Kozeny-Carman para el flujo en la región viscosa, y de la Ecuación de Burke-Plummer para la región turbulenta [6].

$$
\frac{\Delta P}{\rho}=\frac{150(1-\varepsilon)^{2} \mu \nu_{o} L}{g_{c} \varepsilon^{3} \phi^{2} d_{e}^{2} \rho}+\frac{1.75(1-\varepsilon) v_{o}^{2} L}{g_{c} \varepsilon^{3} \phi d_{e}}
$$

donde $\Delta \mathrm{p} / \rho$ es la perdida friccional para el flujo a través del lecho, $v_{0}$ es la porosidad del lecho, vo es la velocidad superficial del aire, L es la altura del lecho, g es un factor de conversión a utilizar con unidades de ingeniería Americanas (no SI $g_{c}=1$ ), $\phi$ es la esfericidad de las partículas, $d_{e}$ es el diámetro equivalente de las partículas y $\rho$ es la densidad del aire.

En la Ecuación (1) encontramos la complicación de encontrar un diámetro equivalente cuando tratamos con el secado industrial de cubos (esfericidad en el inicio del secado $=0.81$ ) o cilindros (esfericidad en el inicio del secado $=0.87$, para $h=d$ ) de frutas o vegetales, mas aun más complicado cuando tenemos que considerar el encogimiento y la pérdida de forma, a lo largo del proceso de secado. Debido al encogimiento de productos alimenticios, la medida de diámetro de la partícula se vuelve extremamente dificil, lo que llevó a RAPOUZAS, DRISCOLL \& SRZEDNICKI [14] a utilizar una ingeniosa modificación de la ecuación de Ergun que implicita el diámetro equivalente, para estudiar la caída de presión en tiras y hojuelas de cebolla, como se puede ver en la Ecuación (2):

$\frac{\Delta P}{L}=A v_{o} \frac{(1-\varepsilon)^{2}}{\varepsilon^{3}}+B v_{o}^{2} \frac{(1-\varepsilon)}{\varepsilon^{3}}$

donde $\Delta \mathrm{P}$ es la caída de presión $(\mathrm{Pa})$ y A y B son constantes empiricas.

Existe muy poca información relacionada con flujo a través de lechos rellenos de partículas de frutas y hortalizas, principalmente considerando el encogimiento que ocurre a lo largo del secado. Uno de los pocos trabajos fue realizado por RATTI [15] que realizó un trabajo extenso de encogimiento y porosidad del lecho de varios productos durante el secado.

E1 objetivo de este trabajo fue estudiar el encogimiento de cubos de papa, remolacha y zanahoria durante el secado y su efecto sobre la porosidad del lecho y la caída de pressión del lecho fixo de $10 \mathrm{~cm}$ de altura. Varias correlaciones fueron utilizadas para describir los cambios de volumen y porosidad del lecho en función del contenido de humedad y relaciones entre caída de presión y velocidad en diferentes contenidos de humedad necesarios en estudios de optimización.

\section{2 - MATERIAL Y METODOS}

\section{1 - Material}

Se utilizaron papas (Solanum tuberosum, L), remolacha (Beta vulgaris, L.) y zanahorias (Daucus carota,
L.) adquiridos en Mercado local y almacenadas en una cámara frigorifica a temperatura de $7^{\circ} \mathrm{C}$ para posteriormente ser usadas.

\section{2 - Preparación del material}

Las muestras fueron peladas manualmente y cortadas con formatos de cubos de aristas de $8 \mathrm{~mm}$, con un equipamiento construido en el departamento de ingeniería y tecnología de alimentos de la UNESP. Las muestras fueron deshidratadas con un secador de bandejas con convección forzada construido por GABAS [3]. Este secador consiste de una columna de $1.02 \mathrm{~m}$ de altura de sección cuadrada, con lado igual a $40 \mathrm{~cm}$, un ventilador centrífugo, conectado a un motor eléctrico monofásico de 220V, 1/3HP y 1750rpm, un sistema de calentamiento del aire compuesto por 8 resistencias eléctricas de hilo níquel-cromo de resistividad 2.122 $\mathrm{ohm} / \mathrm{m}$. La temperatura del aire utilizada para todas las muestras fue de $70^{\circ} \mathrm{C}$, la espesura de la camada fue de 0.10 metros y la velocidad del aire fué de $1.0 \mathrm{~m} / \mathrm{s}( \pm 0.002$ $\mathrm{m} / \mathrm{s})$ y el tiempo de secado fue variado para obtenerse productos con varias humedades.

\section{3 - Medidas de encogimiento}

Para determinar el volumen de particulas experimentalmente, al final del proceso de secado, se ha hecho el recubrimiento del material poroso (principalmente en bajas humedades) con parafina (densidad $0.923 \mathrm{~g} / \mathrm{mL}$ ), y posteriormente fue seguida la metodologia de desplazamiento de tolueno según ZOGZAS [21]. Los volúmenes fueron determinados en duplicado. La diferencia entre estos valores nunca fue mayor de 4.3\%. Muestras del mismo proceso de secado fueran utilizadas para la determinación de humedad por secado en estufa a $105^{\circ} \mathrm{C}$.

El volumen de lecho de partículas fue determinado por la medida de la altura multiplicando por el área de la sección transversal de un conteiner. La mayor diferencia encontrada entre los duplicados de estas experiencias fue de $3.6 \%$.

La porosidad del lecho fue obtenida a través de su definición:

$\varepsilon=\frac{\left(V_{L}-V_{r}\right)}{V_{L}}$

donde $\mathrm{V}_{\mathrm{L}}$ es el volumen del lecho e $\mathrm{V}_{\mathrm{r}}$ es el volumen real ocupado por las partículas en el lecho (que es la suma de los volúmenes de los cubos en el lecho).

\section{4 - Unidad modular para determinar caída de presión}

E1 esquema de la unidad utilizada para medir caída de presión experimental es representado en la Figura 1. Esta consiste de tres partes básicas - sistema de controle del caudal de aire, sección de calentamiento y columna de medida de caída de presión, todo equipado con un sistema de control basado en tecnologia Fieldbus fornecida por la SMAR industria de equipamientos limitada. Como indicado en la Figura 1, el soplador 
centrifugo (1) fue utilizado para forzar el aire a través del lecho relleno. E1 soplador fue controlado por un motor eléctrico con el caudal controlado por un modulador de frecuencia (2) (modelo Siemens MMV). Un medidor de orificio (3) conectado a un transmisor de presión (SMAR, modelo LD302) (4) fue instalado después del ventilador para medir el caudal durante las medidas de caída de presión. Antes de la cámara de caída de presión fue instalado un sistema de flujo de aire uniformizado (8), constituido por 68 tubos de cobre $(\mathrm{DE}=12 \mathrm{~mm}$, espesura $1.5 \mathrm{~mm}$ ), soldados entre si, constituyendo una colmena y ocupando toda la sección transversal del conducto vertical. La cámara de caída de presión (10) consiste de una columna de $0.18 \mathrm{~m}$ de diámetro con $1.04 \mathrm{~m}$ de altura. Fue instalado un tubo de pitot (9) conectado a un transmisor de presión (SMAR, modelo LD302) (11) entre el sistema de flujo uniformizado (8) y la cámara de caída de presión (10) con el objetivo de conferir la velocidad del aire en la entrada. La temperatura del flujo de aire (6) fue medida on-line utilizando transmisores de temperatura (SMAR, modelo TT302). Fueron instalados cinco puntos $(1=0.006 \mathrm{~m}, 2=0.01 \mathrm{~m}$, $3=0.016 \mathrm{~m}, 4=0.02 \mathrm{~m}, 5=0.026 \mathrm{~m}$ ) a lo largo de la columna, para las medidas de presión estática con transmisores de presión (SMAR, modelo LD302) (10), para poder realizar estudios en función de la espesura del lecho. En este trabajo fue fijado un lecho de espesura de $0.01 \mathrm{~m}$ entre los puntos 2 e 4 por medio de telas metálicas y las medidas utilizadas para la caída de presión fueron realizadas en los puntos 1 y 5 . De manera a integrar los instrumentos con el sistema Fieldbus, fue utilizado un convertidor de corriente de 4 a $20 \mathrm{~mA}$ (SMAR, modelo IF302) (13). La operación del sistema fue operada y controlada por medio de un computador (14) utilizando el software AIMAXÒ-WIN.

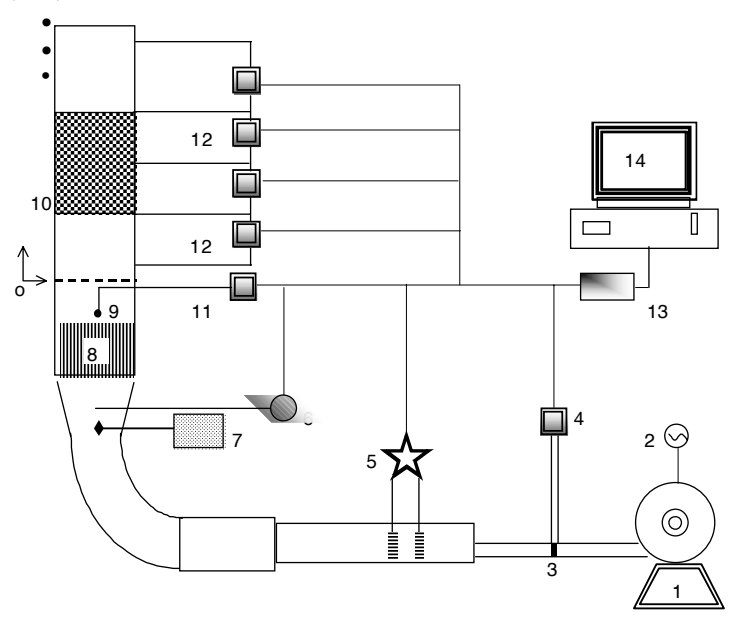

FIGURA 1. Equipamiento experimental para determinar caída de presión: 1. Ventilador Centrifugo, 2. Modulador de frecuencia, 3. Placa de orificio, 4. Transmisor de Presión, 5. Resistencias eléctricas, 6. Temperatura de bulbo seco, 7. Higrómetro, 8. Sistema de flujo uniformizado, 9. Tubo de Pitot, 10. Cámara de secado 11. Transmisora de presión para medir velocidad 12. Transmisores de presión para medir caída de presión 13. Conversor de potencia, 14. Computadora.
Las medidas de caída de presión experimentales (12) fueron realizadas en lechos de cubos de zanahoria, papa y remolacha, pré-secadas, de espesura de $0.01 \mathrm{~m}$, velocidades superficiales del aire 0.1, 0.2, 0.3, 0.7, 0.9, $1.0,1.3,1.5,1.7 \mathrm{~m} / \mathrm{s}( \pm 0.002 \mathrm{~m} / \mathrm{s})$. Para cada medida de velocidad superficial fueron triplicadas las medidas de caída de presión para encontrar el desvío de la medida de presión. El secado durante estos experimentos pudo ser considerado despreciable.

\section{3 - RESULTADOS Y DISCUSIONES}

\section{1 - Variación de volumen de cubos de papa, remolacha y zanahoria}

La variación del volumen de los cubos de papa, remolacha y zanahoria está presentada en la Figura 2. También en esta figura pudimos observar la dispersión de los datos, principalmente de la zanahoria y notar que el volumen de todos los productos disminuye con la humedad, hasta una relación X/Xo entre 0.30 e 0.45 .

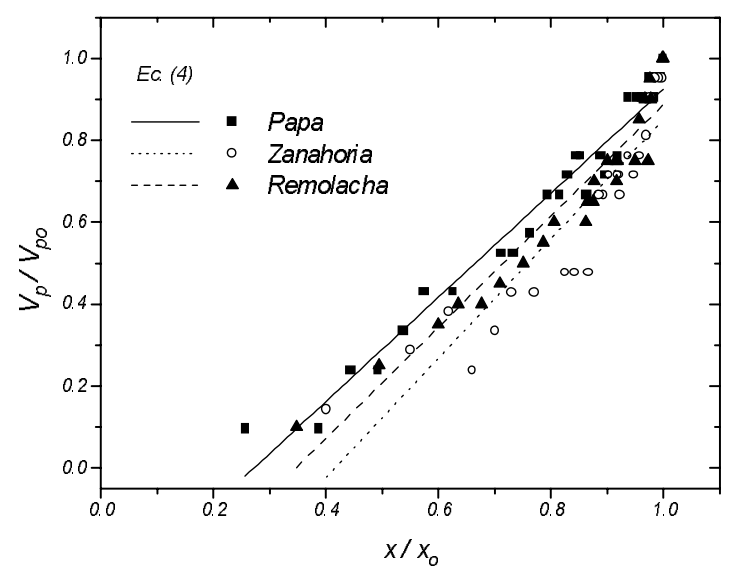

FIGURA 2. Volumen de cubos de papa, remolacha y zanahoria en función de la humedad adimensional del producto.

Estos resultados están de acuerdo con varios resultados encontrados en la literatura $[4,5,7,8,11,12,16$, $17,18,20,21]$.

Observase que de manera general, el volumen final fue de cerca de $10 \%$ del volumen inicial, típico del proceso de secado en condiciones medianas-altas de temperatura $[8,18]$.

El encogimiento de los cubos puede ser representado por la Ecuación (4), que es basada en el principio de la adición de los volúmenes del sólido y del agua que componen los productos. Los parámetros de la Ecuación (4), calculados por regresión linear están presentados en la Tabla 1.

$$
\frac{V_{P}}{V_{P o}}=A_{P}+B_{P} \frac{X}{X_{o}}
$$

donde : $V_{p}$ es el volumen de los cubos $\left(\mathrm{m}^{3}\right), \mathrm{V}_{\mathrm{po}}$ es el volumen inicial de los cubos en $\left(\mathrm{m}^{3}\right), \mathrm{X}$ es el contenido 
de humedad en base seca, $X_{o}$ es el contenido de humedad inicial en base seca.

TABLA 1. Parámetros de la Ecuación 4, calculados por regresión linear.

\begin{tabular}{cccc}
\hline Producto & $\mathrm{A}_{\mathrm{P}}$ & $\mathrm{B}_{\mathrm{P}}$ & $\mathrm{R}^{2}$ \\
\hline Papa & -0.35 & 1.27 & 0.96 \\
Zanahoria & -0.61 & 1.45 & 0.85 \\
Remolacha & -0.46 & 1.30 & 0.91 \\
\hline
\end{tabular}

\section{2 - Variación de volumen del lecho de cubos de papa, remolacha y zanahoria}

E1 volumen del lecho relativo al volumen del lecho inicial $\left(\mathrm{V}_{\mathrm{L}} / \mathrm{V}_{\mathrm{LO}}\right)$ fue graficado contra la humedad adimensional media del lecho $\left(\mathrm{X} / \mathrm{X}_{\mathrm{O}}\right)$. La Figura 3 presenta los datos para cubos de papa, remolacha y zanahoria. E1 adimensional de volumen de la camada, obtenido experimentalmente para cada producto en varias humedades también demostró ser función linear de la humedad adimensional, representado por la Ecuación (5).

donde : $\mathrm{V}_{\mathrm{L}}$ es el volumen del lecho de cubos $\left(\mathrm{m}^{3}\right), \mathrm{V}_{\mathrm{Lo}}$ es el volumen inicial del lecho de cubos en $\left(\mathrm{m}^{3}\right)$, $\mathrm{X}$ es el contenido de humedad en base seca, $\mathrm{X}_{\mathrm{o}}$ es el contenido de humedad inicial en base seca.

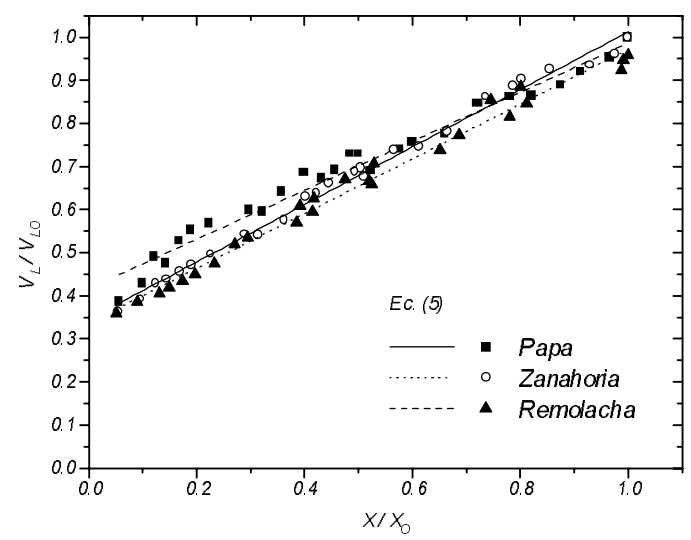

FIGURA 3. Variación del volumen adimensional del lecho de cubos de papa, remolacha y zanahoria.

Los parámetros de la Ecuación (5), calculados por regresión linear están presentados en la Tabla 2.

La reducción de volumen fue linear debido al encogimiento de los cubos, y no hubo problema de acomodamiento del lecho, una vez que las velocidades utilizadas en este estudio fueron abajo de la velocidad de minima fluidización. No fue medida la velocidad de mínima fluidización, porque mismo que las telas metálicas impedian la formación de un lecho fluidizado no se observó este proceso visualmente.
TABLA 2. Parámetros de la Ecuación (5), calculados por regresión linear.

\begin{tabular}{cccc}
\hline Producto & $\mathrm{A}_{\mathrm{L}}$ & $\mathrm{B}_{\mathrm{L}}$ & $\mathrm{R}^{2}$ \\
\hline Papa & 0.41 & 0.57 & 0.98 \\
Zanahoria & 0.34 & 0.67 & 0.99 \\
Remolacha & 0.34 & 0.64 & 0.99 \\
\hline
\end{tabular}

\section{3 - Porosidad del lecho de los cubos de papa, zanahoria y remolacha}

Los cambios de porosidad con la humedad son mostrados en la Figura 4. De manera general, la porosidad aumenta con la disminución de la humedad, de manera mas drastica en baja humedad. Esto ocurrió porque en muy baja humedad, las particulas se vuelven mas deformes debido al encogimiento del propio producto. Estas curvas son similares a las obtenidas por RATTI [15] para lechos de cilindros e discos de papa.

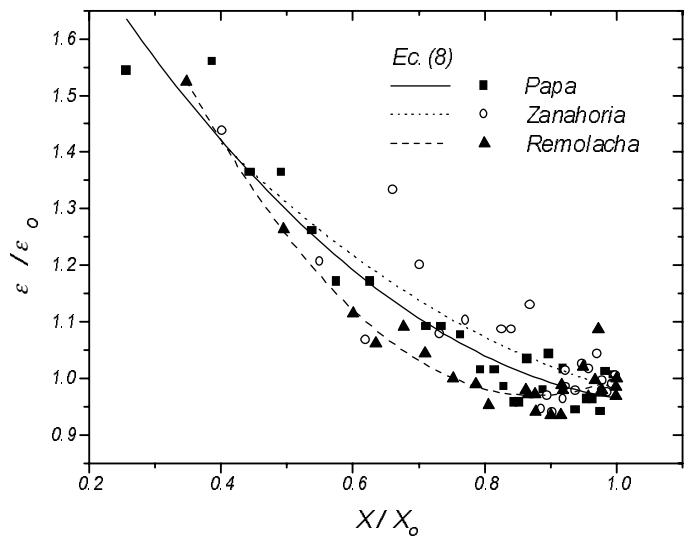

FIGURA 4. Porosidad de cubos de papa, remolacha y zanahoria.

La porosidad no es muy fácil de ser correlacionada por medio de bases teóricas o empíricas. En este trabajo fueron testadas las funciones desarrolladas para densidad real por LOZANO, ROTSTEIN \& URBICAIN [8], Ecuación (6) y NICOLETI et al. [13], Ecuación 7. Ninguna de las dos ecuaciones representó correctamente la porosidad, y por esta razón, optamos por una ecuación parabólica, Ecuación 8. La representación de la Ecuación (8) esta mostrada en la Figura 4.

$\frac{\varepsilon}{\varepsilon_{0}}=C+D\left(\frac{X}{X_{0}}\right)-E \exp \left(-F \frac{X}{X_{0}}\right)$

$\frac{\varepsilon}{\varepsilon_{0}}=C+D\left(\frac{X}{X_{0}}\right)-E\left(\frac{X}{X_{0}}\right) \exp \left(-F \frac{X}{X_{0}}\right)$

$\frac{\varepsilon}{\varepsilon_{0}}=C+D\left(\frac{X}{X_{0}}\right)+E\left(\frac{X}{X_{0}}\right)^{2}$

donde: $\varepsilon$ es la porosidad del lecho, $\varepsilon_{\mathrm{o}}$ es la porosidad inicial del lecho, $X$ es el contenido de humedad en base seca, $X_{0}$ es el contenido de humedad en base seca. 
Las constantes de la Ecuación (8), ajustadas para porosidad de papa, remolacha y zanahoria son presentadas en la Tabla 3.

TABLA 3. Parámetros de la Ecuación 8, calculados por regresión não-linear.

\begin{tabular}{lcccc}
\hline Producto & $\mathrm{C}$ & $\mathrm{D}$ & $\mathrm{E}$ & $\mathrm{R}^{2}$ \\
\hline Papa & 2.10 & -2.04 & 0.92 & 0.94 \\
Remolacha & 1.85 & -1.42 & 0.54 & 0.74 \\
Zanahoria & 2.51 & -3.54 & 2.03 & 0.95 \\
\hline
\end{tabular}

\section{4 - Caída de presión}

Los resultados de caída de presión en función de la velocidad superficial fueron ajustados para cada contenido de humedad, con la ecuación propuesta por RAPOUZAS, DRISCOLL \& SRZEDNICKI [14]. La temperatura utilizada en las pruebas de caída de presión estuvo en un rango de 30 a $32^{\circ} \mathrm{C}$ y con un rango de contenido de humedad relativa de 41 a $45 \%$, de tal manera que la viscosidad y la densidad del aire puedan ser consideradas constantes.

Los coeficientes A e B y el $\mathrm{R}^{2}$ del modelo modificado de Ergun (Ecuación 2) para cada condición fueron obtenidos por análisis de regresión no-linear de los datos experimentales. Estos valores son mostrados para papa, remolacha y zanahoria en la Tabla 4. Los valores de $\mathrm{R}^{2}$ para cada experimento son mayores que 0.94, y los desvios estuvieron entre 1.7 a $5.1 \%$. Por esta razón la ecuación de Ergun modificada fue coherente para representar los resultados de caída de presión en la faja de velocidades estudiadas.

TABLA 4. Coeficientes A e B do modelo de Ergun modificado (Ecuación 2) para lecho de cubos de papa, zanahoria y remolacha.

\begin{tabular}{ccccc}
\hline $\begin{array}{c}\text { Contenido de } \\
\text { humedad } \\
\text { (b.s.) }\end{array}$ & Porosidad & $\begin{array}{c}\mathrm{A} \\
\left(\mathrm{kgm} / \mathrm{m}^{3} \mathrm{~s}\right)\end{array}$ & $\begin{array}{c}\mathrm{B} \\
\left(\mathrm{kgm} / \mathrm{m}^{4}\right)\end{array}$ & $\mathrm{R}^{2}$ \\
\hline Papas & 0.572 & 5.88 & 302.81 & 0.98 \\
4.8 & 0.554 & 8.81 & 310.71 & 0.99 \\
3.5 & 0.572 & 11.15 & 332.31 & 0.97 \\
2.4 & 0.580 & 14.28 & 351.89 & 0.98 \\
1.9 & 0.692 & 12.87 & 427.18 & 0.99 \\
0.9 & 0.556 & 44.21 & 323.47 & 0.99 \\
Zanahoria & 0.631 & 65.42 & 343.08 & 0.96 \\
4.8 & 0.621 & 69.58 & 353.80 & 0.95 \\
3.3 & 0.688 & 74.14 & 365.21 & 0.97 \\
2.4 & 0.683 & 81.87 & 383.78 & 0.98 \\
1.8 & 0.564 & 56.54 & 318.91 & 0.97 \\
1.0 & 0.564 & 63.49 & 337.96 & 0.99 \\
Remolacha & 0.580 & 69.58 & 353.80 & 0.95 \\
4.7 & 0.582 & 74.14 & 365.21 & 0.95 \\
3.5 & 0.736 & 84.72 & 390.40 & 0.98 \\
2.4 & & & & \\
1.8 & & &
\end{tabular}

En la Figura 5 es mostrado que la caída de presión en un lecho de $0.01 \mathrm{~m}$ de partículas aumento con la disminución de porosidad. Esto se debe probablemente a que en una dada velocidad superficial, la menor cantidad de espacios vacios, ofrece mayor resistencia causada por las partículas, resultando en mayor fricción, el cual disipa mas presión.

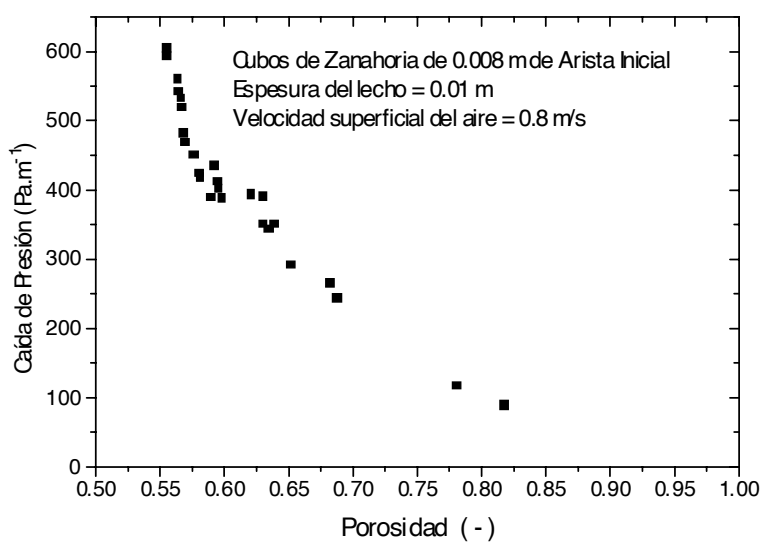

FIGURA 5. Caída de presión en función de la porosidad de un lecho de zanahoria.

De lo ya analizado podemos indicar por la Figura 6 que el modelo de Ergun modificado predice bien la caída de presión de los tres productos en el intervalo de velocidad estudiado. La utilización de la ecuación de Ergun modificada elimina la necesidad de dividir el flujo de aire en laminar o turbulento y determina los coeficientes sin necesidad de medir el diámetro equivalente en función de la humedad. Pero, es sempre necessário, conocer la porosidad del lecho debido el encogimiento de los produtos, es decir, hasta el momento, no es possible predecir la caída de pression sin conocer el volumen del producto en un dado momento.
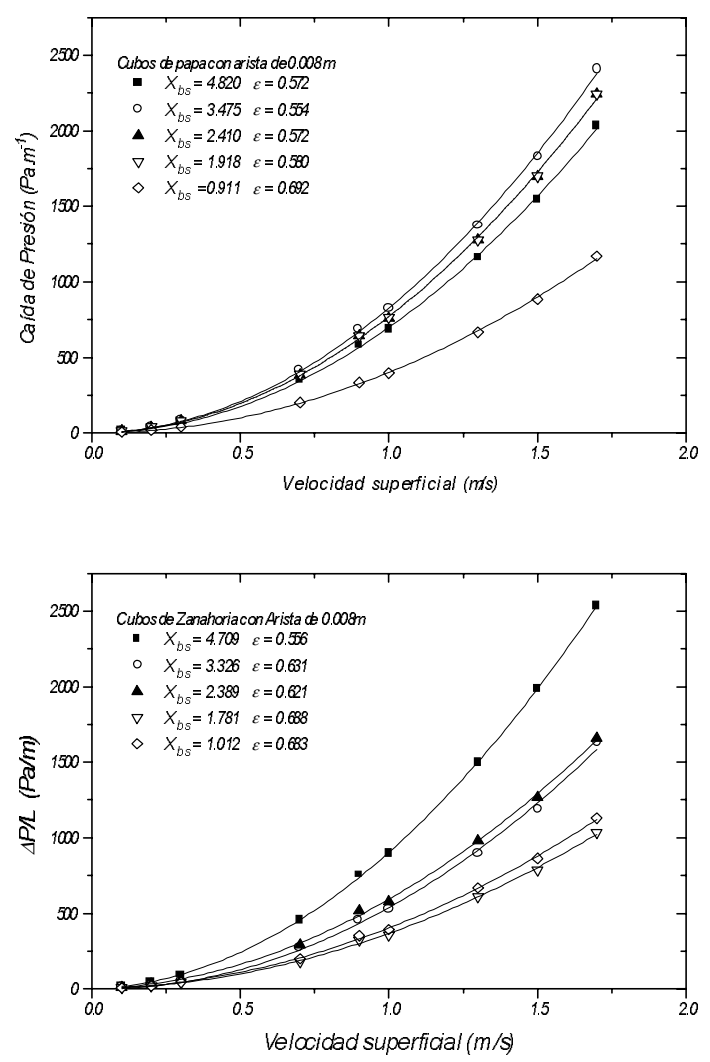


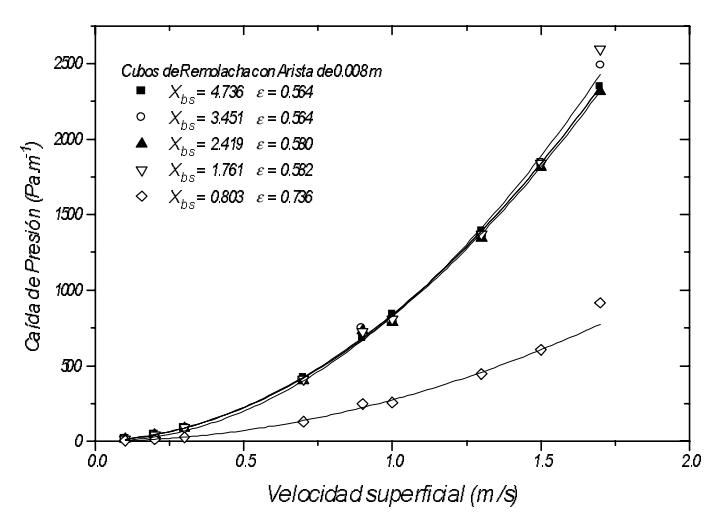

FIGURA 6. Caída de presión de lechos de papas (arriba), zanahoria (medio) y remolacha (abajo) en función de la velocidad superficial del aire.

\section{4 - CONCLUSIONES}

El encogimiento de los productos durante el secado provoca una importante variación de la porosidad de los respectivos lechos, de manera mas importante en el fin del secado, es decir, en bajas humedades. Consecuentemente, ocurre una reducción de la caída de presión durante el secado y este comportamiento no puede ser representado por la clásica ecuación de Ergun, con los datos iniciales del proceso. Una ecuación de Ergun modificada fue empleada satisfactoriamente para representar la caída de presión en los lechos de papas, zanahoria y remolacha.

La reducción de los volúmenes de cubos de vegetales y de sus lechos fue bien representada por una ecuación linear empírica, basada en el principio de la aditividad de volúmenes, mientras para representar bien la porosidad de los lechos fue necesaria el empleo de una ecuación parabólica.

\section{5 - REFERENCIAS}

[1] AKEREDOLU. Shrinkage in dehydration of Manihot utilissima and Dioscorea rotunda. Drying Technology, 5, 107-128, 1987.

[2] CHEN Y CHEN. Effects of dehydration on volume contraction in mushrooms. J. Agric. Eng. Res., 19, 97-99, 1974.

[3] GABAS, A.L. Secagem de Uva Itália em Leito Fixo. MS Thesis, UNICAMP - Campinas - S.P. Brasil, 137 p., 1998.

[4] KHRAISHEH, M.A.M.; COOPER T.J.R.; MAGEE T.R.A.A. Shrinkage characteristics of potatos dehydrated under combined microwave and convective air conditions. Drying Technology, 15, 1003-1022, 1997.

[5] KROKIDA M.K.; MAROULIS Z.B. Effect of drying method on shrinkage and porosity. Drying Technology, 15, 2441-2458, 1997.

[6] LEVENSPIEL, O. Engineering Flow and Heat Exchange, Plenum Press, New York, 373 p., 1993.
[7] LOZANO J.E.; ROTSTEIN E.; URBICAIN M.J. Total porosity and open-pore porosity in the drying of fruits. Journal of Food Science, 45, 1403-1407, 1980.

[8] LOZANO J.E.; ROTSTEIN E.; URBICAIN M.J. Shrinkage, porosity and bulk density of foodstuffs at changing moisture content. Journal of Food Science, 48, 14971502, 1983.

[9] MADAMBA, P.S.; DRISCOLL R.H.; BUCKLE, K.A. Shrinkage, density and porosity of garlic during drying. Journal of Food Engineering, 23, 309-319, 1994.

[10] MCMinN W.A.M.; MAGEE T.R.A. Air drying kinetics of potato cylinders. Drying Technology, 14, 20252040, 1996.

[11] MCMinn W.A.M.; MAGEE T.R.A. Physical characteristics of dehydrated potatoes - Part I. Journal of Food Engineering, 33, 37-48, 1997a.

[12] MCMINN W.A.M.; MAGEE T.R.A. Quality and physical structure of a dehydrated starch-based system. Drying Technology, 15, 1961-1971, 1997b.

[13] NiCOLETI, J.F.; TELIS-ROMERO, J.; TELIS, V.R.N. Air-drying of fresh and osmotically pre-treated pineapple slices: fixed air temperature versus fixed slice temperature drying kinetics. Drying Technology, 19(9): 2175-2191, 2001.

[14] RAPOUZAS, R.S. ; DRISCOLL, R.H. ; SRZEDNICKI, G.S. Bulk Density and Resistance to Airflow of Sliced Onions. Journal of Food Engineering, 26, 67-80, 1995.

[15] RATTI, C. Shrinkage During Drying of Foodstuffs. Journal of Food Engineering, 23, 91-105, 1994.

[16] SARAVACOS G.D.; CHARM S.E. Effect of surface-active agents on the dehydration of fruits and vegetables. Food Technology, 16, 91-93, 1962.

[17] SARAVACOS G.D.; RAOUZEOS G.S. Diffusivity of moisture in air-drying of raisins; In: Mujumdar A. (Ed.), Drying 86, v.2, 487-493, Hemisphere Publishing, New York, 1986.

[18] SOBRAL, P.J.A.; LEBERT, A.; BIMBENET, J.J. Effet du séchage sur le rétrécissement de cubes de pomme de terre. Science des Aliments, 21(3), 231-242, 2001.

[19] SUZUKI K.; KUBOTA K., HASEGAWA T.; HOSAKA H., Shrinkage in dehydration of root vegetables. Journal of Food Science, 41, 1189-1193, 1976.

[20] WANG N.; BRENNAN J.G. Changes in structure, density and porosity of potato during dehydration. Journal of Food Engineering, 24, 61-76, 1995.

[21] ZOGZAS, N.P.; MAROULIS, Z.B.; MARINOS-KOURIS, D. Densities, shrinkage and porosity of some vegetables during air drying. Drying Technology, 12(7), 16531666,1994

\section{6 - AGRADECIMIENTOS}

Los autores agradecen a la Fundação de Amparo à Pesquisa do Estado de São Paulo, FAPESP, proceso No. 98/08738-7, por el soporte financiero, a la SMAR Industrial de Equipamentos Limitada por el soporte técnico, y a la Profa. Dra. Ana Lúcia Gabas del Departamento de Zootecnia de la Faculdade de Zootecnia e Engenharia de Alimentos de la Universidade de São Paulo (USP) por las discusiones y sugestiones durante la realización de este trabajo. 